\title{
Application of Business Intelligence for Business Process Management
}

\author{
Nenad Stefanovic ${ }^{1}$, Dusan Stefanovic ${ }^{2}$, and Milan Misic ${ }^{3}$
}

\begin{abstract}
Companies require highly automated business process management (BPM) functionality, with the flexibility to incorporate business intelligence (BI) at appropriate stages throughout the workflow. Business Activity Monitoring (BAM) unifies these two technologies and provides real-time access to critical performance indicators to improve the speed and effectiveness of business operations. This paper discusses BPM technologies in the context of the supply chain and presents the comprehensive BAM solution that utilizes latest BPM, BI and portal technologies in order to enable decision makers to access and assimilate the right information to make well-informed, timely decisions.
\end{abstract}

\section{Introduction}

Hammer [1] defines a business process as a complete set of end-to-end activities that together create value for the customer. Business Process Management (BPM) is the ability to orchestrate and control the execution of such processes across heterogeneous systems [2].

Business Process Management is the next step in a three-tier environment. Business logic and business rules, now encapsulated in the business logic tier, is extracted from the business logic tier and is presented in a workflow-based environment, which shows graphically the different steps of a business process. At each node, business rules are used to select the next node and business logic is executed. As a consequence, the business rules have become explicit, visible, and

\footnotetext{
${ }^{1}$ Nenad Stefanovic, MSc

Zastava Automobiles, Trg Topolivaca 4, Kragujevac, Serbia email: stefanovic.n@gmail.com

2 Prof. Dusan Stefanovic

University of Kragujevac, Radoja Domanovica 12, Serbia email: dusans@kg.ac.yu

3 Prof. Milan Misic

Higher Technical School, Zvecan, Serbia email: miskekm@eunet.rs
}

Please use the following format when citing this chapter:

Stefanovic, N., Stefanovic, D. and Misic, M., 2008, in IFIP International Federation for Information Processing, Volume 276; Artificial Intelligence and Practice II; Max Bramer; (Boston: Springer), pp. 445449. 
rapidly changeable. This allows a company to react more quickly on changes in the marketplace where it operates.

When BPMS (Business Process Management System) is used to solve business problems, the processing within those solutions is often a black box, making it very difficult for business users and technical support personnel to get a view into what is happening.

On the other hand, Business Intelligence (BI) tools rely on warehouses and data marts while event notification packages track business indicators, yet there's been little focus on merging data in real-time and from warehouses to track the enterprise's lifelines, maximize efficiency, and provide decision support data in context. This is where Business Activity Monitoring comes in. BAM enables any messaging or business process to be fully instrumented, monitored and analyzed in terms that any end-user can understand.

\section{Business Activity Monitoring}

Most, companies have no active real-time element to their BI systems. The consequences are that nothing is helping the business to automatically respond immediately when problems occur or opportunities arise. Also, there is no automatic notification or flagging of alerts to take action that may avoid unnecessary costs, business disruption, operational mistakes and unhappy customers in the future.

Business Activity Monitoring (BAM) is a collection of tools that allow you to manage aggregations, alerts, and profiles to monitor relevant business metrics (Key Performance Indicators - KPIs). It gives users end-to-end visibility into business processes, providing accurate information about the status and results of various operations, processes, and transactions so they can address problem areas and resolve issues within your business. BAM software products incorporate concepts from - and sometimes are built on - ERP, business intelligence, BPM and enterprise application integration (EAI) software.

The BAM provides an easy, real-time, transaction-consistent way to monitor heterogeneous business applications, and to present data for SQL queries and aggregated reports (OLAP). Through queries and aggregations BAM systems can include not only the data that is present during the running business process, but also the state and the dynamics of the running business process, independent of how the business is automated.

BAM applies operational business intelligence and application integration technologies to automated processes to continually refine them based on feedback that comes directly from knowledge of operational events [3]. In addition to auditing business processes (and business process management systems), BAM can send event-driven alerts that can be used to alert decision makers to changes in the business that may require action. 


\section{Example of the BPM solution}

The need for automation and interaction of business processes necessitate the use of modern technologies for managing business process, trading partner relationships and monitoring and analyzing in real-time. For these purposes we have designed two specialized web portals - Business Activity Services (BAS) and Business Activities Monitoring (BAM) portal. This section presents the basis of the comprehensive BPM solution implemented in the automotive company.

\subsection{Business Activity Services}

Business Activity Services (BAS) provides an interaction and collaboration selfservice portal Web site among supply network trading partners.

BAS provides the infrastructure to capture business user input into a business process easily. Then based on the human input, the business process (defined and automated as BPMS orchestration) can continue with the subsequent steps in the pre-defined workflow.

BAS web portal architecture consists of the following modules:

Business User Portal. The self-service Web site that enables business users to interact with partners and business processes through familiar metaphors such as Mailboxes.

Trading Partner Management (TPM). A set of interactive tools and forms that enable the business user to manage online interactions with trading partners. Figure 1 shows web page for Partner Profiles.

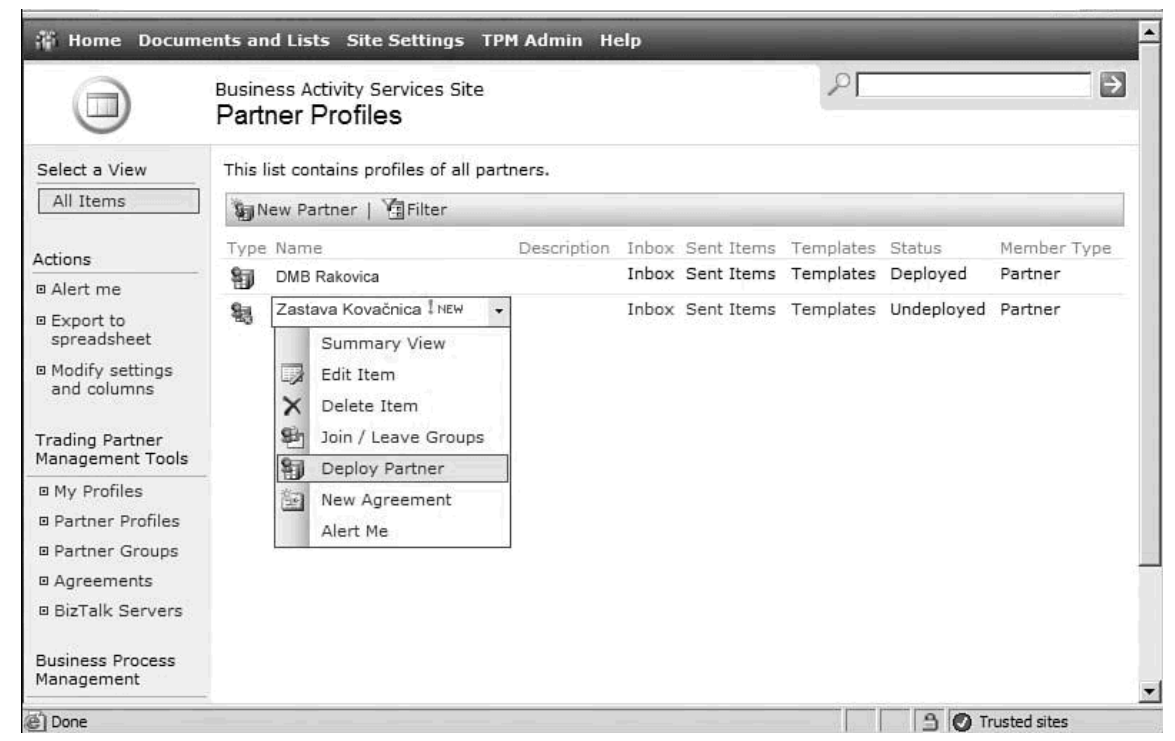

Figure 1 BAS Portal 
Business Process Configuration. This primarily includes the design and programming of the Orchestrations and TPM elements on the BAS site in such a way that the business users can interact with them.

Business User Interaction and Collaboration (with partners and processes). As soon as the orchestrations and TPM elements go through the configuration process by using the key parameters, the business users can use the end-to-end infrastructure to perform the daily interactions with the trading partners.

\subsection{BAM portal}

Business users can use BAM portal to gain a real-time holistic view of business processes that span heterogeneous applications.

There are two ways information workers can use BAM to view business processes: using the spreadsheet application and through BAM web portal.

Each view gives a different perspective on a business process. For example, a BAM view might provide graphical depictions of per-product sales trends or current inventory levels or other key performance indicators. The information in these views might be updated every day, every hour, or more frequently.

Each BAM view relies on one or more BAM activities. A BAM activity represents a specific business process, such as handling purchase orders or shipping a product, and each one has a defined set of milestones and business data. For example, a purchase order activity might have milestones such as Approved, Denied, and Delivered along with business data like Customer Name and Product.

The following list describes how other ways information workers can use BAM features [4]:

- View a single activity instance such as a purchase order or loan (process) in real-time or as historical data.

- Search for activity instances based on their progress or business data (Figure 2).

- Browse aggregations (which are key performance indicators) around all the business activities that are currently being processed or have already happened.

- Navigate to the related activity instances such as shipments associated with given purchase order, or the Invoice in which it is included.

Additionally, it is possible to create different activity-related alerts. Alerts allow us to define important events about business processes, such as Key Performance Indicators (KPIs) that can be delivered to users on a real-time basis. Users subscribe to alerts to receive notification of the business event that the alert monitors. There are two types of alerts, aggregate and instance. An aggregate alert allows specifying threshold data across a time frame whereas an instance alert is based on specific qualifying data points. 


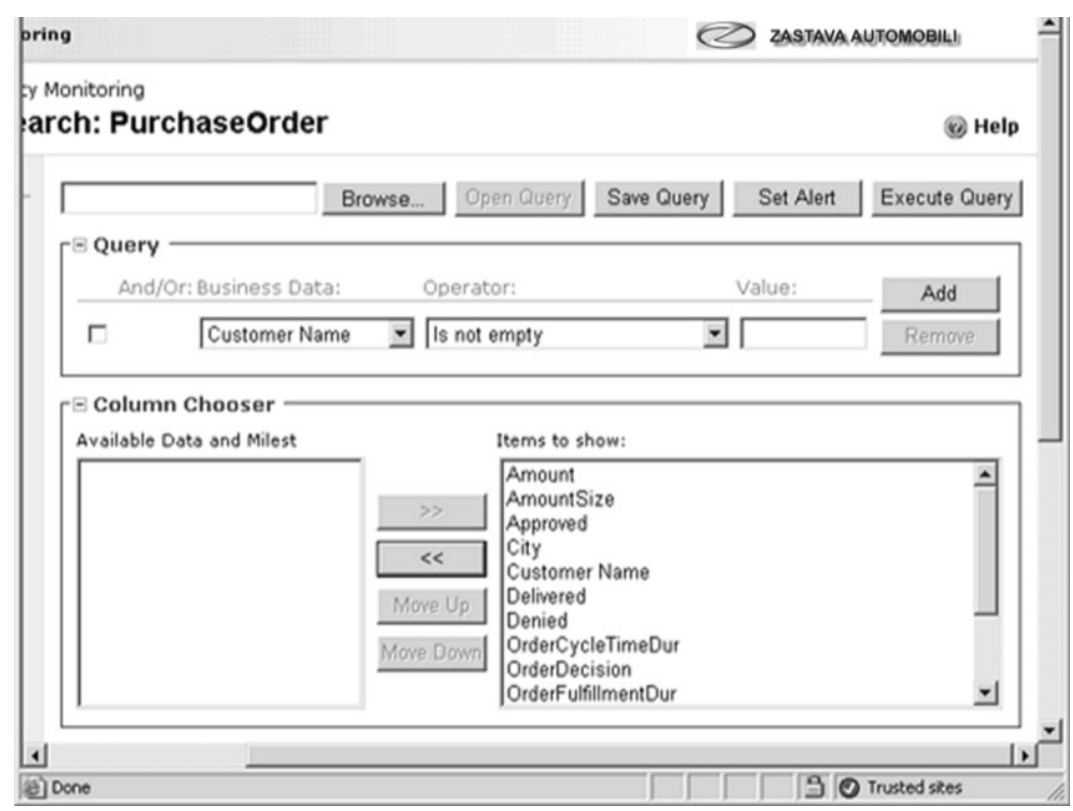

Figure 2 BAM search interface

\section{Conclusion}

This paper introduced the concept of Business Activity Monitoring and highlighted how it can be used to collect real-time information about business processes implemented through the BPMS.

The presented BAM portal provides a rich view into data collected via BAS system and enables activity data to be searched and viewed in a variety of ways. Because the BAM data is held in database tables and views, it's easy to access the information from a variety of tools, including different reporting tools, which can produce a highly detailed tracking portal providing very rich business intelligence.

With BPM and BAM systems in place, all parties in a supply chain network can track the real-time flow of goods, money, and information across the network.

\section{References}

[1] Hammer M, (1996) Beyond Reengineering: how the process-centered organization is changing our work and our lives. London: Harper Collins Business.

[2] Cutlip R, Telford R (2002) The Orchestration of Business Processes, Web Services Journal. 2(6): 28-34.

[3] Microsoft, (2006) What is BAM? http://msdn2.microsoft.com/en-us/library/aa560139.aspx.

[4] Stefanovic N, Stefanovic D (2006) Methodology for BPM in Supply Networks. 5th CIRP ICME, Ischia, Italy. 